\title{
The Use of Vibration Signals for Structural Health Monitoring, System Identification, Test Planning/Optimization, and Dynamic Model Validation/Updating
}

\author{
Samuel da Silva, ${ }^{1}$ Michael Todd, ${ }^{2}$ John S. Sakellariou, ${ }^{3}$ and Maryam Ghandchi-Tehrani ${ }^{4}$ \\ ${ }^{1}$ Departamento de Engenharia Mecânica, Faculdade de Engenharia de Ilha Solteira, Universidade Estadual Paulista (UNESP), \\ Ilha Solteira, SP, Brazil \\ ${ }^{2}$ Department of Structural Engineering, University of California, San Diego, San Diego, CA, USA \\ ${ }^{3}$ Department of Mechanical Engineering \& Aeronautics, Stochastic Mechanical Systems \& Automation Laboratory, \\ University of Patras, Patras, Greece \\ ${ }^{4}$ Engineering and the Environment, University of Southampton, Highfield, Southampton, UK
}

Correspondence should be addressed to Samuel da Silva; samuel@dem.feis.unesp.br

Received 6 September 2016; Accepted 6 September 2016

Copyright (c) 2016 Samuel da Silva et al. This is an open access article distributed under the Creative Commons Attribution License, which permits unrestricted use, distribution, and reproduction in any medium, provided the original work is properly cited.

Vibration signals are naturally available and can be readily measured from many types of engineering systems through a variety of low-cost data acquisition systems and sensors under normal operating or imposed excitation conditions. Based on the appropriate processing of the collected signals, rich information for the structural dynamics may be extracted and used for linear and nonlinear modeling, structural health monitoring, active vibration control, modal analysis, and further system insight that may lead to the design of improved performance, sustainability, safety, and comfort. The aim of this special issue is to collect studies in the state of the art for the abovementioned scientific areas with applications in engineering and preference given to actual systems and numerical and experimental procedures.

$\mathrm{X}$. Wei et al. investigated the use of improved ensemble empirical mode decomposition based on singular value decomposition for filtering chaotic vibration signals in order to reduce noise. The superiority of the proposed method was demonstrated with simulated signal, two-degree-of-freedom chaotic vibration signals, and the experimental signals based on double potential well theory. F. Liu et al. proposed and tested a novel feature called phase space similarity for health monitoring of bearings. Based on vibration signals and this feature, a fault pattern recognition algorithm was performed to predict the remaining useful life estimation. E. J. Diehl and
J. Tang presented an approach to the modeling and analysis of a benchmark two-stage gearbox test bed to characterize gear fault signature when processed with harmonic wavelet transform analysis. The results of the tests have the potential to render the fault detection and diagnosis for gearbox systems easier.

W. Wang et al. used acoustic emission to investigate the process of damage evolution in T700/6808 composite under tensile loading. The main damage contained was the matrix cracking and interface damage as well as the fiber breakage.

A. Concha and L. Alvarez-Icaza estimated the shear model of seismically excited, torsionally coupled building using acceleration data measured considering noisy and corrupt measurements of the ground and floors. A filtering strategy was used to eliminate the disturbance and to attenuate noise. C. G. Rodriguez et al. developed a nonlinear model with two-dimensional three degrees of freedom to evaluate motion under off-design operation conditions. With this model, a limit for springs failure was determined to evaluate whether it is necessary to replace springs or not. C. Xie et al. presented nonlinear aeroelastic models including geometrical nonlinearities, steady and unsteady nonplanar aerodynamics computations, and a structural model using finite element method. Those two aerodynamic models share the same aero mesh and allow the aerogrids to follow up 
with structural deflections via surface spline interpolation between structural and aerogrids. A wind tunnel test was conducted to validate and update this theoretical analysis framework, and reasonable agreement was obtained.

With these papers, the readers can enjoy different approaches in the use of vibration signals processing, damage detection, and identification for structural dynamics and engineering.

Samuel da Silva

Michael Todd

John S. Sakellariou

Maryam Ghandchi-Tehrani 


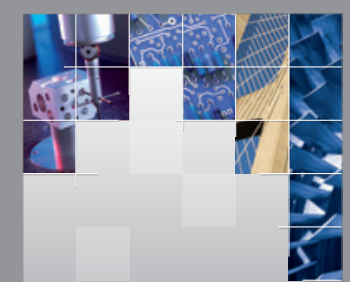

\section{Enfincering}
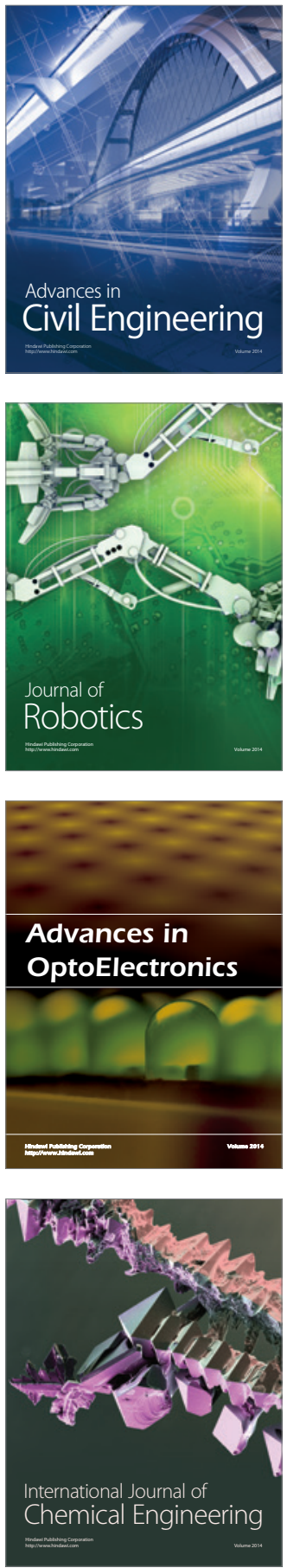

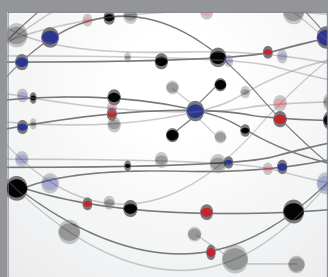

The Scientific World Journal

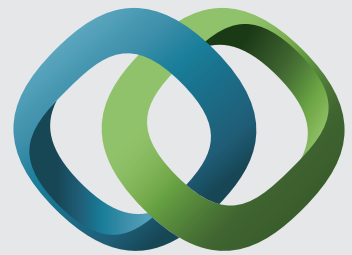

\section{Hindawi}

Submit your manuscripts at

http://www.hindawi.com
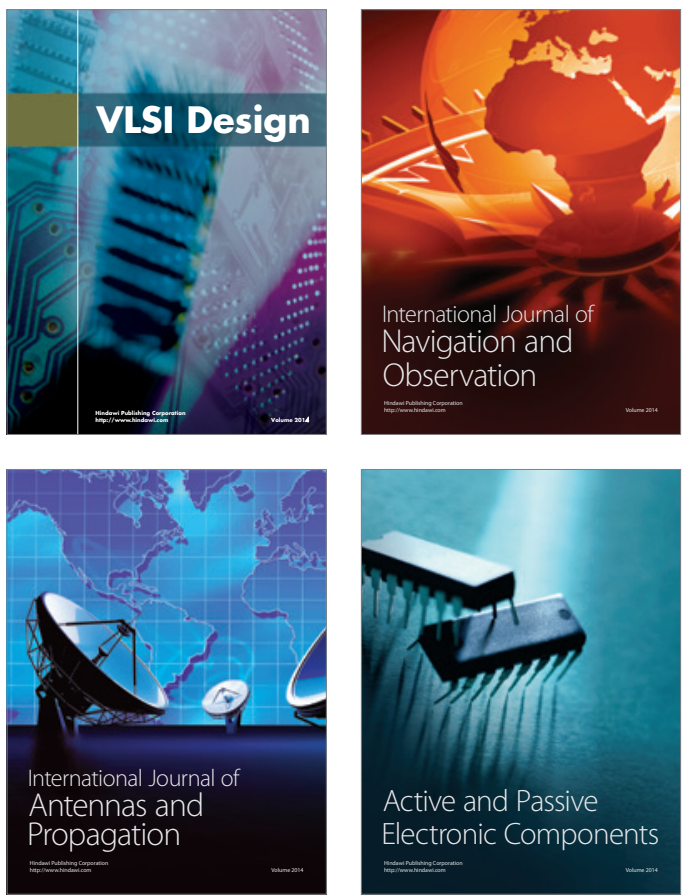
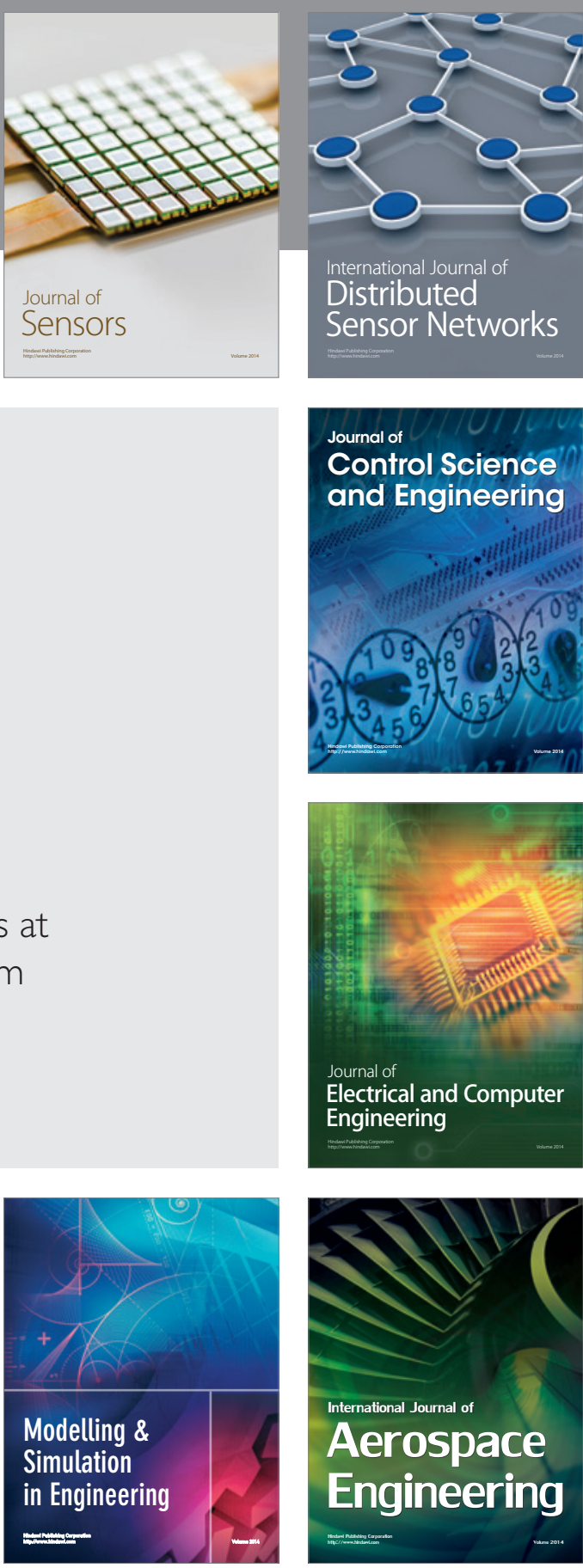

International Journal of

Distributed

Sensor Networks

Journal of

Control Science

and Engineering
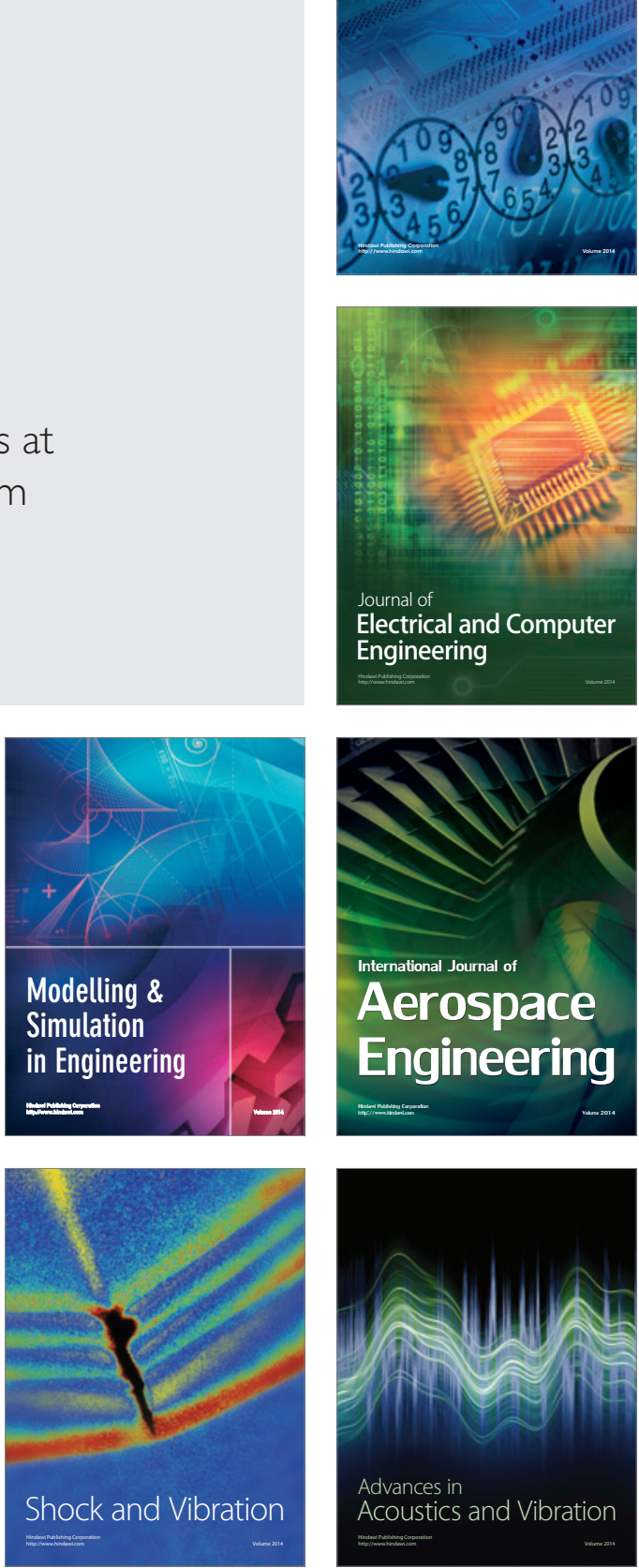